\title{
Question Answering for Dutch using Dependency Relations ${ }^{\star}$
}

\author{
Gosse Bouma, Jori Mur, Gertjan van Noord, Lonneke van der Plas, and Jörg \\ Tiedemann \\ Rijksuniversiteit Groningen \\ g. bouma@rug.nl \\ http://www.let.rug.nl/ gosse/Imix
}

\begin{abstract}
Joost is a question answering system for Dutch which makes extensive use of dependency relations. It answers questions either by table look-up, or by searching for answers in paragraphs returned by IR. Syntactic similarity is used to identify and rank potential answers. Tables were constructed by mining the CLEF corpus, which has been syntactically analyzed in full.
\end{abstract}

\section{Introduction}

Joost is a monolingual QA system for Dutch which makes heavy use of syntactic information. Most questions are answered by retrieving relevant paragraphs from the document collection, using keywords from the question. Next, potential answers are identified and ranked using a number of clues. Apart from obvious clues, we also use syntactic structure to identify and rank answer strings. A second strategy is based upon the observation that certain question types can be anticipated, and the corpus can be searched off-line for answers to such questions. Whereas previous approaches have used regular expressions to extract the relevant relations, we use patterns of dependency relations. To this end, the whole corpus has been analyzed syntactically.

In the next section, we describe the building blocks of our QA system. In section 3, we describe Joost. In section 4, we discuss the results of Joost on the CLEF 2005 QA task.

\section{Preliminaries}

Syntactic Preprocessing. The Alpino-system is a linguistically motivated, wide-coverage, grammar and parser for Dutch in the tradition of HPSG. It consists of over 500 grammar rules and a large lexicon of over 100.000 lexemes.

\footnotetext{
* This research was carried out as part of the research program for Interactive Multimedia Information Extraction, IMIX, financed by NWO, the Dutch Organisation for Scientific Research.
} 
Heuristics have been implemented to deal with unknown words and ungrammatical or out-of-coverage sentences (which may nevertheless contain fragments that are analyzable). The grammar provides a 'deep' level of syntactic analysis. The output of the system is a dependency graph. [1] shows that the accuracy of the system, when evaluated on a test-set of 500 newspaper sentences, is over $88 \%$, which is in line with state-of-the-art systems for English.

Alpino includes heuristics for recognizing proper names. For the QA task, named entity classification was added. To this end, we collected lists of person names $(120 \mathrm{~K})$, geographical names $(12 \mathrm{~K})$, organization names $(26 \mathrm{k})$, and miscalleneous items $(2 \mathrm{~K})$. The data are primarily extracted from the Twente News Corpus, a collection of over 300 million words of newspaper text, which comes with relevant annotation. For unknown names, a maximum entropy classifier was trained, using the Dutch part of the shared task for CONLL 2003. ${ }^{1}$ The accuracy on unseen CONLL data of the resulting classifier (which combines dictionary look-up and a maximum entropy classifier) is $88.2 \%$.

The Dutch text collection for CLEF was tokenized and segmented into (4.1 million) sentences, and parsed in full. We used a Beowulf Linux cluster of 128 Pentium 4 processors $^{2}$ to complete the process in about three weeks. The dependency trees are stored as XML.

Reasoning over Dependency Relations. Several researchers have attempted to use syntactic information, and especially dependency relations, in QA [2-5]. We have implemented a system in which dependency patterns derived from the question must be matched by equivalent dependency relations in a potential answer. The dependency analysis of a sentence gives rise to a set of dependency relations of the form $\langle\mathrm{Head} / \mathrm{HIx}, \mathrm{Rel}$, Dep/DIx $\rangle$, where Head is the root form of the head of the relation, and Dep is the head of the dependent. Hix and DIx are string indices, and Rel the dependency relation. For instance, the dependency analysis of sentence (1-a) is (1-b).

$$
\begin{aligned}
& \text { a. Mengistu kreeg asiel in Zimbabwe (Mengistu was given asylum in } \\
& \text { Zimbabwe) } \\
& \text { b. }\left\{\begin{array}{ll}
\langle\mathrm{krijg} / 2, \text { su, mengistu/1 }\rangle, & \langle\mathrm{krijg} / 2, \text { obj1, asiel } / 3\rangle, \\
\langle\mathrm{krijg} / 2, \bmod , \text { in } / 4\rangle, & \langle\text { in/4, obj1, zimbabwe/5 }\rangle
\end{array}\right\}
\end{aligned}
$$

A dependency pattern is a set of (partially underspecified) dependency relations:

$$
\{\langle\mathrm{krijg} / \mathrm{K}, \text { obj1, asiel/A }\rangle,\langle\mathrm{krijg} / \mathrm{K}, \mathrm{su}, \mathrm{Su} / \mathrm{S}\rangle\}
$$

A pattern may contain variables, represented here by (words starting with) a capital. A pattern $P$ matches a set of dependency relations $R$ if $P \subset R$, under some substitution of variables.

\footnotetext{
${ }^{1}$ http://cnts.uia.ac.be/conll2003/ner/

2 which is part of the High-Performance Computing centre of the University of Groningen
} 
Equivalences can be defined to account for syntactic variation. For instance, the subject of an active sentence may be expressed as a PP-modifier headed by door (by) in the passive:

(3) Aan Mengistu werd asiel verleend door Zimbabwe (Mengistu was given asylum by Zimbabwe)

The following equivalence accounts for this:

$\{\langle\mathrm{V} / \mathrm{I}, \mathrm{su}, \mathrm{S} / \mathrm{J}\rangle\} \Leftrightarrow\{\langle$ word $/ \mathrm{W}, \mathrm{vc}, \mathrm{V} / \mathrm{I}\rangle,\langle\mathrm{V} / \mathrm{I}, \bmod , \operatorname{door} / \mathrm{D}\rangle,\langle\operatorname{door} / \mathrm{D}$, obj $1, \mathrm{~S} / \mathrm{J}\rangle\}$

Here, the verb word is (the root form of) the passive auxiliary, which takes a verbal complement headed by the verb $\mathrm{V}$.

Given an equivalence $L h s \Leftrightarrow R h s$, substitution of Lhs in a pattern $P$ by $R h s$ gives rise to an equivalent pattern $P^{\prime}$. A pattern $P$ now also matches with a set of relations $R$ if there is some equivalent pattern $P^{\prime}$, and $P^{\prime} \subset R$, under some substitution of variables.

We have implemented 13 additional equivalence rules, to account for, among others, word order variation within appostions, the equivalence of genitives and van-PPs, equivalence between appositions and simple predicative sentence, coordination, and relative clauses. In [6], we show that the inclusion of equivalence rules has a positive effect on various components of our QA system.

Off-line Retrieval. Off-line methods have proven to be very effective in QA [7]. Before actual questions are known, a corpus is exhaustively searched for potential answers to specific question types (capital, abbreviation, year of birth, ...). The answers are extracted from the corpus off-line and stored in a structured table for quick and easy access.

[8] show that extraction patterns defined in terms of dependency relations are more effective than regular expression patterns over surface strings. Following this observation, we used the module for dependency pattern matching to exhaustively search the parsed corpus for potential answers to frequently occurring question types. For instance, the pattern in (4) extracts information about organizations and their founders.

$$
\{\langle\text { richt_op/R, su, Founder } / S\rangle,\langle\text { richt_op/R, obj1, Founded } / 0\rangle\}
$$

The verb oprichten (to found) can take on a wide variety of forms (active, with the particle $o p$ split from the root, participle, and infinitival, either the founder or the organization can be the first constituent in the sentence, etc. In all cases, modifiers may intervene between the relevant constituents:

(5) a. Minderop richtte de Tros op toen .... (Minderop founded the Tros when...)

b. Kasparov heeft een nieuwe Russische Schaakbond opgericht en... (Kasparov has founded a new Russian Chess Union and...)

The pattern in (4) suffices to extract this relation from both of the examples above. Equivalence rules can be used to deal with other forms of syntactic varia- 


\begin{tabular}{|c|c|c|c|c|c|c|}
\hline Relation & tuples & uniq & Relation & tuples uniq & Relation & tuples \\
\hline Abbreviation & 21.497 & 8.543 & Currency & $\begin{array}{ll}6.619 & 222\end{array}$ & Function & 77.02846 .589 \\
\hline Age & 22.143 & 18520 & Died Age & 1.127834 & ||Inhabitants & 708 \\
\hline Born Date & 2356 & 1.990 & Died Date & $583 \quad 544$ & Nobel Prize & 169 \\
\hline Born Loc & 937 & 879 & Died Loc & $664 \quad 583$ & & \\
\hline Capital & 2.146 & 515 & Founded & 1.021953 & & \\
\hline
\end{tabular}

Table 1. Size of extracted relation tables. Each second and third column list the overall number of extracted tuples and extracted unique tuples (types) respectively.

tion. For instance, once we define a pattern to extract the country and its capital from (6-a), equivalence rules ensure that the alternative formulations in (6-b)(6-c) match as well. Table 1 lists all the relations we extracted.

(6) a. de hoofdstad van Afghanistan, Kabul (the capital of Afghanistan, Kabul)

b. Kabul, de hoofdstad van Afghanistan (Kabul, the capital of Af'stan)

c. Afghanistans hoofdstad, Kabul (Af'stan's capital, Kabul)

Extracting ISA relations. Fine-grained named entity classification based on a.o. labels obtained from appositions, is useful for answering wH-questions and definition questions $[9,10]$. From the fully parsed Dutch CLEF text collection, we extracted $602 \mathrm{~K}$ unique apposition tuples, consisting of a noun (used as class label) and a named entity. The resulting table contains, for instance, 112 names of ferry boats (Estonia, Anna Maria Lauro, Sally Star etc.) and no less than 2951 national team coaches (Bobby Robson, Jack Charlton, Menotti, Berti Vogts etc.). By focussing on the most frequent label for a named entity, most of the noise can be discarded. For instance, Guus Hiddink occurs 17 times in the extracted apposition tuples, 5 times as bondscoach (national team chef), and once with various other labels (boss, colleague, guest, newcomer, ...). In [11], we show that automatically acquired class labels for named entities improve the performance of our QA system on which questions and definition questions.

\section{Joost}

In this section, we describe the components of our QA system, Joost. Depending on the question class, questions are answered either by table look-up, or by a combination of IR and linguistic techniques. Potential answers are ranked on the basis of a score which combines, among others, IR-score, frequency of the answer, and the amount of overlap in dependency relations between question and the sentence from which the answer was extracted.

Question Analysis. Each incoming question is parsed by Alpino. To improve parsing accuracy on this specific task, the disambiguation model was retrained on a corpus which contained annotated and manually corrected depen- 
dency trees for 650 quiz questions. ${ }^{3}$ For CLEF 2005, we used a model which was trained on data which also included (manually corrected dependency trees of) the CLEF 2003 and 2004 questions. It achieved an accuracy of 97.6 on CLEF 2005 questions.

On the basis of the dependency relations returned by the parser the question class is determined. Joost distinguishes between 29 different question classes. 18 question classes are related to the relation tuples that were extracted offline. Note that a single relation can often be questioned in different ways. For instance, whereas a frequent question type asks for the meaning of an acronym (What does the abbreviation RSI stand for?), a less frequent type asks for the abbreviation of a given term (What is the abbreviation of Mad Cow Disease?). The other 11 question classes identify questions asking for an amount, the date or location of an event, the (first) name of a person, the name of an organization, how-questions, WH-questions, and definition questions.

For each question class, one or more syntactic patterns are defined. For instance, the following pattern accounts for questions asking for the capital of a country:

$$
\left\{\begin{array}{lc}
\langle\text { wat } / \mathrm{W}, \text { wh }, \text { is } / \mathrm{I}\rangle, & \langle\text { is } / \mathrm{I}, \text { su, hoofdstad } / \mathrm{H}\rangle \\
\langle\text { hoofdstad/H, mod, van/V }\rangle, & \langle\text { van/V, obj1, Country/C }\rangle
\end{array}\right\}
$$

Depending on the question class, it is useful to identify one or two additional arguments . For instance, the dependency relations assigned to the question Wat is de hoofdstad van Togo? (What is the capital of Togo?) match with the pattern in (7), and instantiate Country as Togo. Therefore, the question class capital is assigned, with Togo as additional argument. Similarly, Who is the king of Norway? is classified as function(king, Norway), and In which year did the Islamic revolution in Iran start? is classified as date(revolution).

Some question classes require access to lexical semantic knowledge. For instance, to determine that In which American state is Iron Mountain? asks for a location, the systeem needs to know that state refers to a location, and to determine that Who is the advisor of Yasser Arafat? should be classifed as function(advisor, Yasser Arafat), it needs to know that advisor is a function. We obtained such knowledge mainly from Dutch EuroWordNet [12]. The list of function words (indicating function roles such as president, queen, captain, secretary-general, etc.) was expanded semi-automatically with words from the corpus that were distributionally similar to those extracted from EWN (see [11] for details).

Question classification was very accurate for the CLEF 2005 questions. There were a few cases where the additional arguments selected by the system did not seem the most optimal choice. Two clear mistakes were found (e.g. What is the currency of Peru? was classified as currency (of) and not as currency (Peru)).

Information Retrieval. For questions which cannot be answered by the relation tables, traditional keyword-based information retrieval (IR) is used to narrow down the search space for the linguistically informed part of the QA

\footnotetext{
${ }^{3}$ From the Winkler Prins spel, a quiz game made available to us by Het Spectrum, bv.
} 
system which identifies answers. On the basis of keywords from the question, the IR system retrieves relevant passages from the corpus.

Keywords are derived from the question using its content words. Function words and other irrelevant words are removed using a static stop word list. We implemented an interface to seven publicly available IR engines [13]. We selected Zettair [14] as the underlying system in our experiments because of speed and recall performance. The entire CLEF QA corpus (in its tokenized plain text version) has been indexed using the IR engine with its standard setup.

Earlier experiments have shown that a segmentation into paragraphs is most efficient for IR performance in QA. We used the existing markup in the corpus to determine the paragraph boundaries. This resulted in about 1.1 million paragraphs (including headers that have been marked as paragraphs). We did experiments with additional pre-processing, e.g., including proper lemmatization (using Alpino root forms) but we could not improve the IR performance compared to the baseline using standard settings. However, we did include labels of named entities found by Alpino in each paragraph as additional tokens. This makes it possible to search for paragraphs including certain types of named entities (e.g. location names and organizations) and special units (e.g. measure names and temporal expressions) corresponding to question types found by the question analyses component.

For the QA@CLEF 2005 data, we used Zettair to return the 40 most relevant paragraphs for a query. This gives an answer recall (for questions which have an answer in the text collection) of $91 \%$. On average, 4.74 paragraphs are required to find an answer, and half of the answers are found in the top 2 paragraphs.

Answer Identification and Ranking. For questions that are answered by means of table look-up, the relation table provides an exact answer string. For other questions, it is necessary to extract answer strings from the set of paragraphs returned by IR. Given a set of paragraph id's, we retrieve from the parsed corpus the dependency relations for the sentences occurring in these paragraphs.

Various syntactic patterns are defined for (exact) answer identification. For questions asking for the name of a person, organization, or location, or for an amount or date, a constituent headed by a word with the appropriate named entity class has to be found. As all of these occur frequently in the corpus, usually many potential answers will be identified. An important task is therefore to rank potential answers.

The following features are used to determine the score of a short answer A extracted from sentence $\mathrm{S}$ :

- Syntactic Similarity The proportion of dependency relations from the question which match with dependency relations in $\mathrm{S}$.

- Answer Context A score for the syntactic context of A.

- Names The proportion of proper names, nouns, and adjectives from the query which can be found in $\mathrm{S}$ and the sentence preceding $\mathrm{S}$.

- Frequency The frequency of A in all paragraphs returned by IR.

- IR The score assigned to the paragraph from which A was extracted. 
The score for syntactic similarity implements a preference for answers from sentences with a syntactic structure that overlaps with that of the question. Answer context implements a preference for answers that occur in the context of certain terms from the question. Given a question classified as date (Event), for instance, date expressions which occur as a modifier of Event are preferred over date expressions occurring as sisters of Event, which in turn are preferred over dates which have no syntactic relation to Event.

The overall score for an answer is the weighted sum of these features. Weights were determined manually using previous CLEF data for tuning. The highest weights are used for Syntactic Similarity and Answer Context. The highest scoring answer is returned as the answer.

Ranking of answers on the basis of various features was initially developed for IR-based QA only. Answers found by table look-up were ranked only by frequency. Recently, we have started to use the scoring mechanism described above also for answers stemming from table look-up. As the tables contain pointers to the sentence from which a tuple was extracted, we can easily go back to the source sentence, and apply the scoring mechanisms described above. ${ }^{4}$ Using more features to rank an answer provides a way to give the correct answer to questions like Who is the German minister of Economy? The function table contains several names for German ministers, but does not distinguish between different departments. The most frequent candidate is Klaus Kinkel (54 entries), who is minister of foreign affairs. The correct name, Günter Rexrodt, occurs only 11 times. Using Syntactic Similarity and Names as an additional features, Joost manages to give the correct answer.

Special Cases. We did not implement techniques which deal specifically with temporally restricted questions (i.e. Which vulcano erupted in June 1991?). The mechanism for scoring potential answers takes into account the syntactic similarity and the overlap in names (including date expressions) between question and answer sentence, and this implements a preference for answers which are extracted from contexts referring to the correct date. Note that, as the same scoring technique is used for answers found by table look-up, this strategy should also be able to find the correct answer for questions such as Who was the mayor of Moscow in 1994?, for which the function table might contain more than one answer.

General wH-questions, such as (8), are relatively difficult to answer. Whereas for most question types, the type of the answer is relatively clear (i.e. it should be the name of a person or organization, or a date, etc.), this is not the case for wH-questions.

(8) a. Which fruit contains vitamin C?

b. Which ferry sank southeast of the island Utö?

To improve the performance of our system on such questions, we make use of two additional knowledge sources. From EuroWordNet, we imported all hypernym relations between nouns. Question (8-a) is assigned the question class

\footnotetext{
${ }^{4}$ As no IR is involved in this case, the IR score is set to 1 for all answers.
} 
which(fruit). We use the hypernym relations to assign a higher score to answers which are hypernyms of fruit. ${ }^{5}$ As EuroWordNet does hardly include proper names, we also used the ISA-relations extracted from appositions containing a named entity, as described in section 2. Question (8-b) is assigned the question class which (ferry). Candidate answers that are selected by Joost are: Tallinn, Estonia, Raimo Tiilikainen etc. Since, according to our apposition database, Estonia is the only potential answer which ISA ferry, this answer is selected.

CLEF 2005 contained no less than 60 definition questions (i.e. What is Sabena?, Who is Antonio Matarese?). We used the IsA-relations extracted from appositions to answer such questions. More in particular, our strategy for answering definition questions consisted of two phases:

- Phase 1: The most frequent class found for a named entity is selected.

- Phase 2: The sentences which mention the named entity and the class are retrieved and searched for additional information which might be relevant. Snippets of information that are in a adjectival relation or which are a prepositional complement to the class label are selected.

A disadvantage of focussing on class labels is that the label itself is not always sufficient for an adequate definition. Therefore, in phase 2 we expand the class labels with modifiers which typically need to be included in a definition. For the question What is Sabena?, our system produces Belgian airline company as answer.

\section{Evaluation}

The results of the CLEF evaluation are as follows:

\begin{tabular}{|l|r|r|r|}
\hline Question Type & \# questions & \multicolumn{2}{|c|}{ correct answers } \\
& & $\#$ & $\%$ \\
\hline Factoid & 114 & 62 & 54.39 \\
Temporally Restricted Factoid & 26 & 7 & 26.92 \\
Definition & 60 & 30 & 50 \\
\hline Overall & 200 & 99 & 49.5 \\
\hline
\end{tabular}

The scores are satisfactory for factoid questions and definitions. It is unclear to us at the moment what the explanation is for the fact that the system performed less well on temporally restricted questions. Of the 140 factoid questions, 46 questions were assigned a type corresponding to a relation table. For 35 of these questions, an answer was actually found in one of the tables. The other 11 questions were answered by using the IR-based strategy as fall-back. 52 of the 60 definition questions were answered by the strategy described in section 3 .

\footnotetext{
${ }^{5}$ Unfortunately, EuroWordNet only contains two hypernyms for the synset fruit, none of which could be used to identify an answer to (8-a).
} 
For the other definition questions, the general IR-based strategy was used as fall-back. Three definition questions received NIL as an answer.

Parsing errors are the cause of some wrong or incomplete answers. The question Who is Javier Solana?, for instance, is answered with Foreign Affairs, which is extracted from a sentence containing the phrase Oud-minister van buitenlandse zaken Javier Solana (Ex-minister of foreign affairs, Javier Solana). Here, Javier Solana was erroneously analyzed as an apposition of affairs. Similarly, the wrong answer United Nations for the question What is UNEP?, which was extracted from a sentence containing the environment programme of the United Nations (UNEP), which contained the same attachment mistake.

A frequent cause of errors were answers that were echoing (part of) the question. Currently, the system only filters answers which are a literal substring of the question. This strategy fails in cases like Q: Where is Bonn located? A: in Bonn. and Q: In which city does one find the famous Piazza dei Miracoli? A: at the Piazza dei Miracoli. It seems these cases could be easily filtered as well, although in some cases substantial overlap between question and answer does lead to a valid answer (e.g Q: What is the name of the rocket used to launch the satellite Clementine? A: Titan rocket).

Our strategy for answering definition questions seemed to work reasonably well, although it did produce a relatively large number of inexact answers (of the 18 answers that were judged inexact, 13 were answers to definition questions). This is a consequence of the fact that we select the most frequent class label for a named entity, and only expand this label with adjectival and PP modifiers that are adjacent to the class label (a noun) in the corresponding sentence. Given the constituent the museum Hermitage in St Petersburg, this strategy fails to include in St Petersburg, for instance. We did not include relative clause modifiers, as these tend to contain information which is not appropriate for a definition. However, for the question, Who is Iqbal Masih, this leads the system to answer twelve year old boy, extracted from the constituent twelve year old boy, who fought against child labour and was shot sunday in his home town Muritke. Here, at least the first conjunct of the relative clause should have been included.

\section{Conclusion}

We have shown that dependency parsing of both questions and the full document collection is useful for developing an adequate QA system. Dependency patterns can be used to search the corpus exhaustively for answers to frequent question types and for class labels for named entities, which are used to improve the performance of the system on which-questions and definition questions. Selection of the most likely answer to a question uses a syntactic similarity metric based on dependency relations.

We have used a limited number of equivalences over dependency relations. An obvious next step is to expand this set with equivalences derived automatically from the parsed corpus (i.e. as in [15]). The syntactic techniques we employ operate exclusively on individual sentences. In the future, we hope to extend 
this to techniques which operate on the paragraph level by integrating, among others, a component for coreference resolution. In the near future, we also hope to be able to use dependency relations to boost the performance of IR [16].

\section{References}

1. Malouf, R., van Noord, G.: Wide coverage parsing with stochastic attribute value grammars. In: IJCNLP-04 Workshop Beyond Shallow Analyses - Formalisms and statistical modeling for deep analyses, Hainan (2004)

2. Katz, B., Lin, J.: Selectively using relations to improve precision in question answering. In: Proceedings of the workshop on Natural Language Processing for Question Answering (EACL 2003), Budapest, EACL (2003) 43-50

3. Litkowski, K.C.: Use of metadata for question answering and novelty tasks. In Voorhees, E.M., Buckland, L.P., eds.: Proceedings of the eleventh Text Retrieval Conference (TREC 2003), Gaithersburg, MD (2004) 161-170

4. Mollá, D., Gardiner, M.: Answerfinder - question answering by combining lexical, syntactic and semantic information. In: Australasian Language Technology Workshop (ALTW) 2004, Sydney (2005)

5. Punyakanok, V., Roth, D., Yih, W.: Mapping dependency trees: An application to question answering. In: The 8th International Symposium on Artificial Intelligence and Mathematics (AI\&Math 04), Fort Lauderdale, FL (2004)

6. Bouma, G., Mur, J., van Noord, G.: Reasoning over dependency relations for QA. In: Proceedings of the IJCAI workshop on Knowledge and Reasoning for Answering Questions (KRAQ), Edinburgh (2005) 15-21

7. Fleischman, M., Hovy, E., Echihabi, A.: Offline strategies for online question answering: Answering questions before they are asked. In: Proc. 41st Annual Meeting of the Association for Computational Linguistics, Sapporo, Japan (2003) 1-7

8. Jijkoun, V., Mur, J., de Rijke, M.: Information extraction for question answering: Improving recall through syntactic patterns. In: Coling 2004, Geneva (2004) 12841290

9. Pasca, M.: Acquisition of categorized named entities for web search. In: Proceedings of the Thirteenth ACM conference on Information and knowledge management. (2004) $137-145$

10. Pantel, P., Ravichandran, D.: Automatically labeling semantic classes. In Susan Dumais, D.M., Roukos, S., eds.: HLT-NAACL 2004: Main Proceedings, Boston, Massachusetts, USA, Association for Computational Linguistics (2004) 321-328

11. van der Plas, L., Bouma, G.: Automatic acquisition of lexico-semantic knowledge for question answering. In: Proceedings of Ontolex 2005 - Ontologies and Lexical Resources, Jeju Island, South Korea (2005)

12. Vossen, P.: Eurowordnet a multilingual database with lexical semantic networks (1998)

13. Tiedemann, J.: A comparison of off-the-shelf IR engines for question answering. Poster presentation at CLIN 2004, Leiden, The Netherlands (2004)

14. Zobel, J., Williams, H., Scholer, F., Yiannis, J., Hein, S.: The Zettair Search Engine. Search Engine Group, RMIT University, Melbourne, Australia. (2004)

15. Lin, D., Pantel, P.: Discovery of inference rules for question answering. Natural Language Engineering 7 (2001) 343-360

16. Tiedemann, J.: Integrating linguistic knowledge in passage retrieval for question answering. In: Proceedings of EMNLP 2005, Vancouver (2005) 939-946 